\title{
How well do we understand the reaction rate of $\mathrm{C}$ burning?
}

\author{
S. Courtin ${ }^{1,2,3, \star}$, C.L. Jiang ${ }^{4}$, , G. Fruet ${ }^{1,2,}$, K. Auranen ${ }^{4}$, M.L. Avila ${ }^{4}$, A.D. Ayangeakaa ${ }^{4}$, B.B. Back ${ }^{4}$, S. Bottoni ${ }^{4}$, M.
} Carpenter $^{4}$, C. Dickerson ${ }^{4}$, B. DiGiovine ${ }^{4}$, J.P. Greene ${ }^{2}$, D.J. Henderson ${ }^{4}$, C.R. Hoffman ${ }^{4}$, R. V.F. Janssens ${ }^{4}$, B.P. $\mathrm{Kay}^{4}$, S.A. Kuvin ${ }^{4}$, T. Lauritsen ${ }^{4}$, R.C. Pardo ${ }^{4}$, K.E. Rehm ${ }^{4}$, D. Santiago-Gonzalez ${ }^{4}$, J. Sethi ${ }^{4}$, D. Seweryniak ${ }^{4}$, R. Talwar $^{4,}$, C. Ugalde ${ }^{4}$, S. Zhu ${ }^{4}$, C.M. Deibel ${ }^{5}$, S.T. Marley ${ }^{5}$, D. Bourgin ${ }^{1,2}$, F. Haas ${ }^{1,2,}$, M. Heine ${ }^{1,2,}$, D. Montanari ${ }^{1,2,3,}$, D.G. Jenkins ${ }^{6}$, L.G. Morris ${ }^{6}$, A. Lefebvre-Schuhl ${ }^{7}$, S. Almaraz-Calderon ${ }^{8}$, X. Fang ${ }^{9}$, X.D. Tang ${ }^{10}$, M. Alcorta ${ }^{11}$, B. Bucher $^{12,}$, M. Albers $^{4}$, and P. Bertone ${ }^{4}$,

\author{
${ }^{1}$ IPHC, Université de Strasbourg, F-67037 Strasbourg, France \\ ${ }^{2}$ CNRS, UMR7178, F-67037 Strasbourg, France \\ ${ }^{3}$ USIAS, F-67083 Strasbourg, France \\ ${ }^{4}$ Physics Division, Argonne National Laboratory, Argonne, IL 60439, USA \\ ${ }^{5}$ Department of Physics and Astronomy, Louisiana State University, Baton Rouge, LA70803, USA \\ ${ }^{6}$ Department of Physics, University of York, Heslington, York YO10 5DD, UK \\ ${ }^{7}$ CSNSM IN2P3-CNRS and University of Paris Sud, F-91405 Orsay, France \\ ${ }^{8}$ Department of Physics, Florida State University, Tallahassee, FL 32306, USA \\ ${ }^{9}$ University of Notre Dame, Notre Dame, IN 46556, USA \\ ${ }^{10}$ Institute of Modern Physics, Lanzhou, China \\ ${ }^{11}$ TRIUMF, Vancouver, BC V6T 2A3, Canada \\ ${ }^{12}$ Lawrence Livermore National laboratory, Livermore, CA 94551, USA
}

\begin{abstract}
Carbon burning plays a crucial role in stellar evolution, where this reaction is an important route for the production of heavier elements. A particle- $\gamma$ coincidence technique that minimizes the backgrounds to which this reaction is subject and provides reliable cross sections has been used at the Argonne National Laboratory to measure fusion cross-sections at deep sub-barrier energies in the ${ }^{12} \mathrm{C}+{ }^{12} \mathrm{C}$ system. The corresponding excitation function has been extracted down to a cross section of about $6 \mathrm{nb}$. This indicates the existence of a broad S-factor maximum for this system. Experimental results are presented and discussed.
\end{abstract}

\section{Introduction}

Reaction rates for $\mathrm{C}$ burning are essential ingredients to understand the production of chemical elements heavier than carbon as well as the evolution of massive stars. Carbon burning processes determine whether a star will join to the heavy-ion burning branches following hydrogen and helium burning and if white dwarfs will evolve into type Ia supernovae. It is thus of very high importance to know the ${ }^{12} \mathrm{C}+{ }^{12} \mathrm{C}$ fusion cross section with good accuracy from the Coulomb barrier (CB) down to the Gamow window which is centered around $\mathrm{E}_{G}=1.5 \pm 0.3 \mathrm{MeV}$ at a temperature of $\mathrm{T}=5 \times 10^{8} \mathrm{~K}$ [1]. In a stellar environments, $\mathrm{C}$ burning occurs essentially via the ${ }^{12} \mathrm{C}+{ }^{12} \mathrm{C}$ fusion reaction. The exit channels for this reaction are : ${ }^{12} \mathrm{C}\left({ }^{12} \mathrm{C}, \alpha\right){ }^{20} \mathrm{Ne}$, ${ }^{12} \mathrm{C}\left({ }^{12} \mathrm{C}, \mathrm{p}\right){ }^{23} \mathrm{Na}$ and ${ }^{12} \mathrm{C}\left({ }^{12} \mathrm{C}, \mathrm{n}\right){ }^{23} \mathrm{Mg}$. The associated Qvalues are $4.62 \mathrm{MeV}, 2.24 \mathrm{MeV}$ and $-2.62 \mathrm{MeV} \mathrm{MeV}$ respectively. The ${ }^{23} \mathrm{Mg}$ channel with negative Q-value is essentially closed at deep sub-barrier energies.

One of the most striking results obtained in the early studies of heavy-ion collisions is the observation of resonant structures in the reaction cross-sections : i.e. elas-

\footnotetext{
^e-mail: sandrine.courtin@iphc.cnrs.fr
}

tic, inelastic and fusion channels of some light heavy-ion systems. These structures were found especially strong in the fusion cross section of the ${ }^{12} \mathrm{C}+{ }^{12} \mathrm{C}$ system at energies above the $\mathrm{CB}$ down to sub barrier energies. These resonances have often been attributed to ${ }^{12} \mathrm{C}-{ }^{12} \mathrm{C}$ molecular configurations of the ${ }^{24} \mathrm{Mg}$ compound nucleus, and their strength related to the number of open channels in the reaction which is minimal for this system at the $\mathrm{CB}$ [2]. The possible persistance of these resonances at astrophysical energies is still a debated question. For example, the resonance phenomena in ${ }^{12} \mathrm{C}+{ }^{12} \mathrm{C}$ have been explained through the impact on the cross section of the relatively large spacings and the narrow widths of ${ }^{24} \mathrm{Mg}$ compound levels in the corresponding excitation-energy region [3] years. The ${ }^{12} \mathrm{C}+{ }^{12} \mathrm{C}$ fusion reaction has been the object of a realm of experimental investigations in the past [4-10]. The lowest-energy measured resonance in this reaction is at $\mathrm{E}_{\text {c.m. }}=2.1 \mathrm{MeV}$ [9], only partially overlapping with the high-energy part of the Gamow window. Some of the previous experimental results are presented in Fig. 1.

Techniques used to measure these $\mathrm{S}$ factors where based on the identification of charged particles, i.e. $\mathrm{p}$ and $\alpha$, or of the $\gamma$-rays emitted from the evaporation residues, 


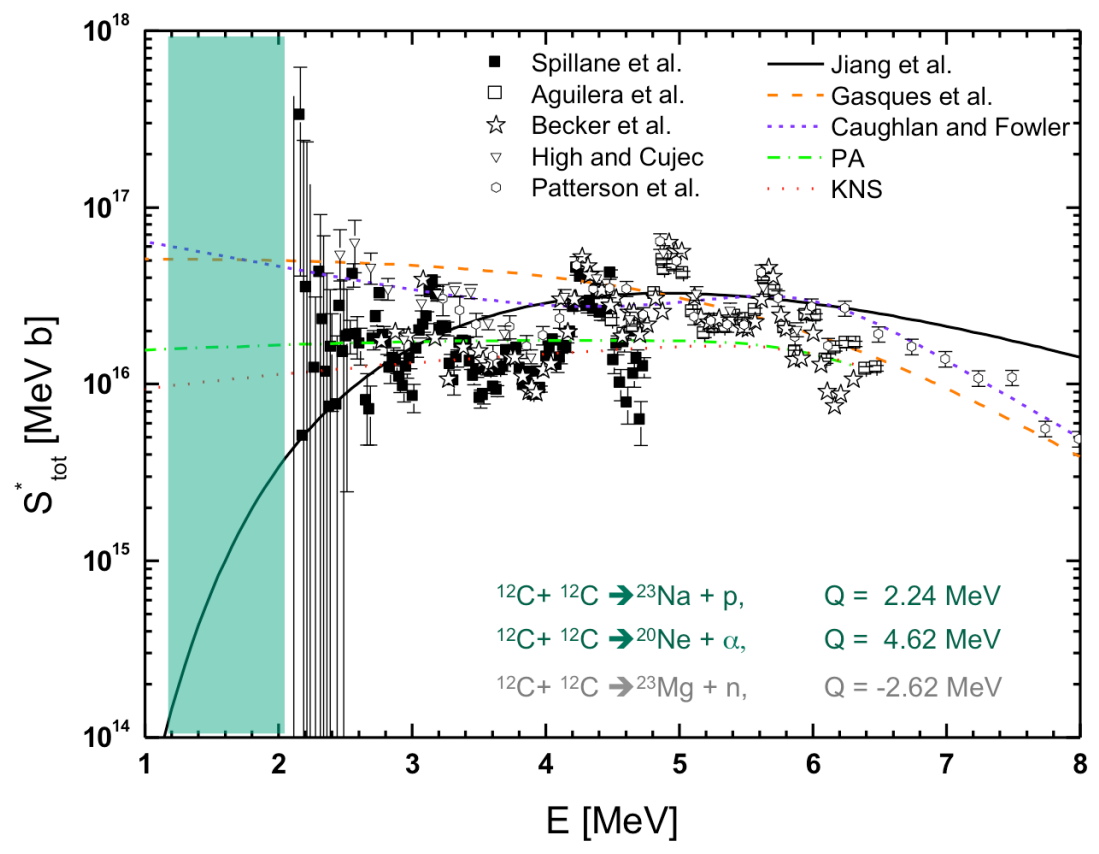

Figure 1. Experimental $\mathrm{S}$ factor for the ${ }^{12} \mathrm{C}+{ }^{12} \mathrm{C}$ fusion reaction from refs. $[4,6,7,9,10]$. The astrophysics region is indicated by the green area.

${ }^{20} \mathrm{Ne}$ and ${ }^{23} \mathrm{Na}$. It should be noted that at the lowest investigated energies, the error bars are large and large discrepancies appear between the different measurements. Interestingly enough, the different extrapolations based on different potentials differ from more than 2 orders of magnitude in the Gamow region. The ubiquitus contamination of helium and deuterium in the target can indeed lead to severe background at low energies for both techniques. Moreover measurements based on $\gamma$-ray detection are subject to room and cosmic $\gamma$ backgrounds. To suppress these backgrounds, a new technique has been developed at the Argonne National Laboratory recently, based on $\gamma$-particle coincidences. Details about the technique as well as spectra describing the drastic suppression of background are given in Ref. [11]. This method was used in the present work.

\section{Experimental set-up}

The experiment has been performed at the Argonne National Laboratory using a ${ }^{12} \mathrm{C}$ beam delivered by the ATLAS facility impinging on a highly enriched $(99.9 \%){ }^{12} \mathrm{C}$ target. The beam intensity was $\sim 600 \mathrm{pnA}$ and the target thickness was $\sim 50 \mu \mathrm{g} . \mathrm{cm}^{-2}$.

Ten energy points have been measured between $\mathrm{E}_{c . m .}=$ 4.93 and $2.68 \mathrm{MeV}$. Gamma transitions from the evaporation residues were measured using the Gammasphere 100 Ge detectors array in coincidence with charged particles (p and $\alpha$ ) from 3 annular double sided silicon detectors. Fig. 2 shows the target chamber with 2 annular detectors at backward angles and 1 at forward angles covering in total $\sim 25 \%$ of $4 \pi$. Normalization of the beam current was obtained using a Faraday cup and two surface barrier Si monitor detectors identifiying scattered ${ }^{12} \mathrm{C}$ nuclei, at $45^{\circ}$ forward angles.

The ${ }^{12} \mathrm{C}\left({ }^{12} \mathrm{C}, \alpha\right){ }^{20} \mathrm{Ne}$ and ${ }^{12} \mathrm{C}\left({ }^{12} \mathrm{C}, \mathrm{p}\right){ }^{23} \mathrm{Na}$ were identified by gating on the characteristic $\gamma$ transitions $1635 \mathrm{keV}$ $2^{+} \rightarrow 0^{+}$of ${ }^{20} \mathrm{Ne}$ and $440 \mathrm{keV} \frac{5}{2}^{+} \rightarrow \frac{3}{2}^{+}$of ${ }^{23} \mathrm{Na}$. The associated $\alpha$ and p particles were identified in coincidence in the annular DSSDs.

The measured cross-sections converted into $\mathrm{S}$ factors are presented in the next section.

\section{Results and discussion}

Figure 3 shows ${ }^{12} \mathrm{C}+{ }^{12} \mathrm{C} \mathrm{S}$ factors as a function of $\mathrm{E}_{c . m}$. measured in the present work in the type IA supernova Gamow energy region (indicated by the yellow region on the figure) together with the most recent results for the same system [12]. The present data, which lowest point corresponds to a cross section of $6 \mathrm{nb}$, is in fairly good agreement with this measurement but shows smaller error bars. It should be noted that at the lowest measured energies, the data seem to indicate a decreasing $\mathrm{S}$ factor, which would be in agreement with the Jiang extrapolation 


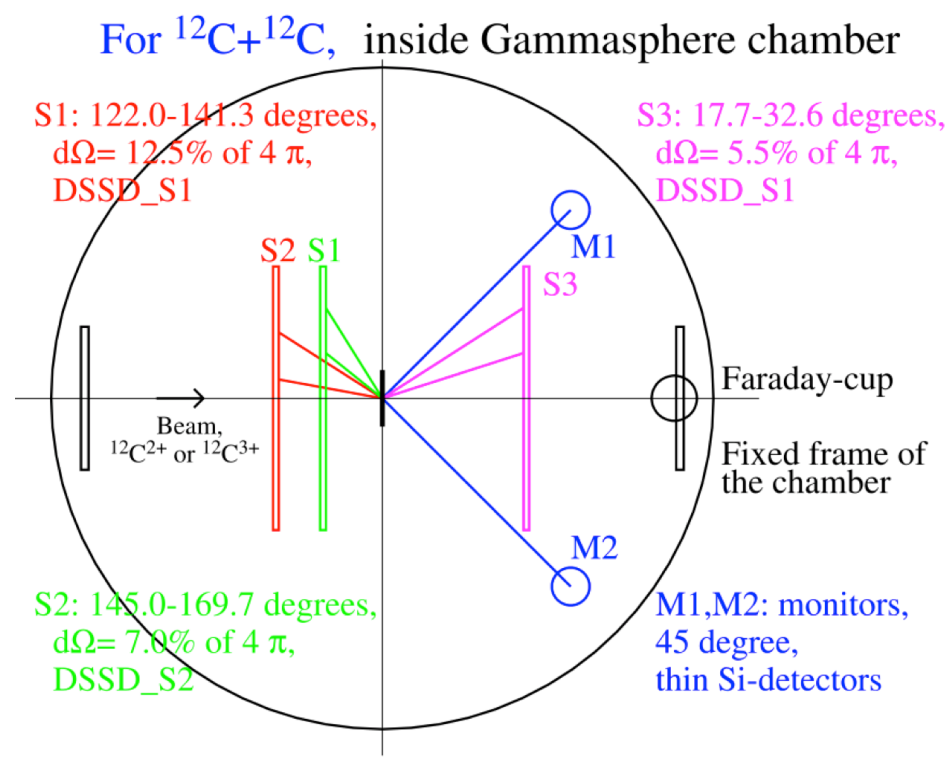

Figure 2. Schematic view of the target chamber showing the 3 annular DSSDs, the Si monitors and the Faraday cup.

[15]. Extrapolations predicting an increasing S factor or a slight decrease at low energies are based on standard potentials and use the sudden model or coupled-channel approach whereas the Jiang extrapolation takes into account the fusion hindrance phenomenon. This phenomenon was introduced by Jiang et al. fifteen years ago in fusion reactions of medium-mass systems, measured below the 0.1 $\mathrm{mb}$ regime [17]. Indeed, at low energies, the fusion cross sections are smaller than predicted by coupled-channel calculations using standard Woods-Saxon potentials. Several theoretical descriptions were proposed for this phenomenon : i. a consequence of the saturation properties of the nuclear matter introduced in calculations using a double-folding potentiel + repulsive core [18], ii. the effects of a 2 steps process involving the capture in a 2 body potential pocket and then the penetration of a one body potential to reach a compound nucleus state [19], iii. Effects of the Pauli repulsion during the fusion process introduced recently by $\mathrm{C}$. Simenel et al. and presented at this Fusion 17 conference.

Two representations have been used to discuss signatures of fusion hindrance, the logarithmic derivative of the energy-weighted cross section and the $\mathrm{S}$ factor. In the latter, often used for systems of astrophysics relevance, a maximum and a decrease of $\mathrm{S}$ when going down in energy is taken as a signature of fusion hindrance. Interestingly enough, the present data seem to be in agreement with the presence of a broad $\mathrm{S}$ factor maximum. This phenomenon may have dramatic consequences on the reaction rate of $\mathrm{C}$ burning and the subsequent nucleosynthesis of heavier chemical elements in massive stars.

\section{Acknowledgements}

This work was supported by the US Department of Energy, Office of Nuclear Physics, under Contract No. DE-AC0206CH11357 and by USIAS, Strasbourg Fellowship of S. Courtin.

\section{References}

[1] C. E. Rolfs, and W. S. Rodney, Cauldrons in the Cosmos, The University of Chicago Press, 1988.

[2] F. Haas and Y. Abe, Phys. Rev. Lett. 46 (1981).

[3] C.L. Jiang et al., Phys. Rev. Lett. 110072701 (2013).

[4] Patterson L J et al., The Astrophys. J. 157367 (1969).

[5] Mazarakis M et al. Phys. Rev. C 71280 (1973).

[6] High M D and Cujec B, Nucl. Phys. A 282181 (1977).

[7] H.W. Becker et al., ZPA 303, 305312 (1981).

[8] Barron-Palos L R et al., Nucl. Phys. A 779218 (2006).

[9] Aguilera E F et al., Phys. Rev. C 73064 (2006).

[10] Spillane T et al., Phys. Rev. Lett. 98122 (2007).

[11] C.L. Jiang et al., NIMA 682, 1215 (2012).

[12] J. Zickefoose, PhD Thesis, U. Connecticut, (2011) .

[13] W. Fowler, G. Caughlan and B. Zimmerman, Annu. Rev. Astrophys. 13, 69 (1975).

[14] L.R. Gasques et al., Phys. Rev.. C 72, 025806 (2005).

[15] C.L. Jiang, K.E. Rehm, B.B. Back and R.V.F. Janssens, Phys. Rev. C 75, 015803 (2007), and references therein.

[16] H. Esbensen, X.D. Tang, and C.L. Jiang, Phys. Rev. C 84, 064613 (2011). 


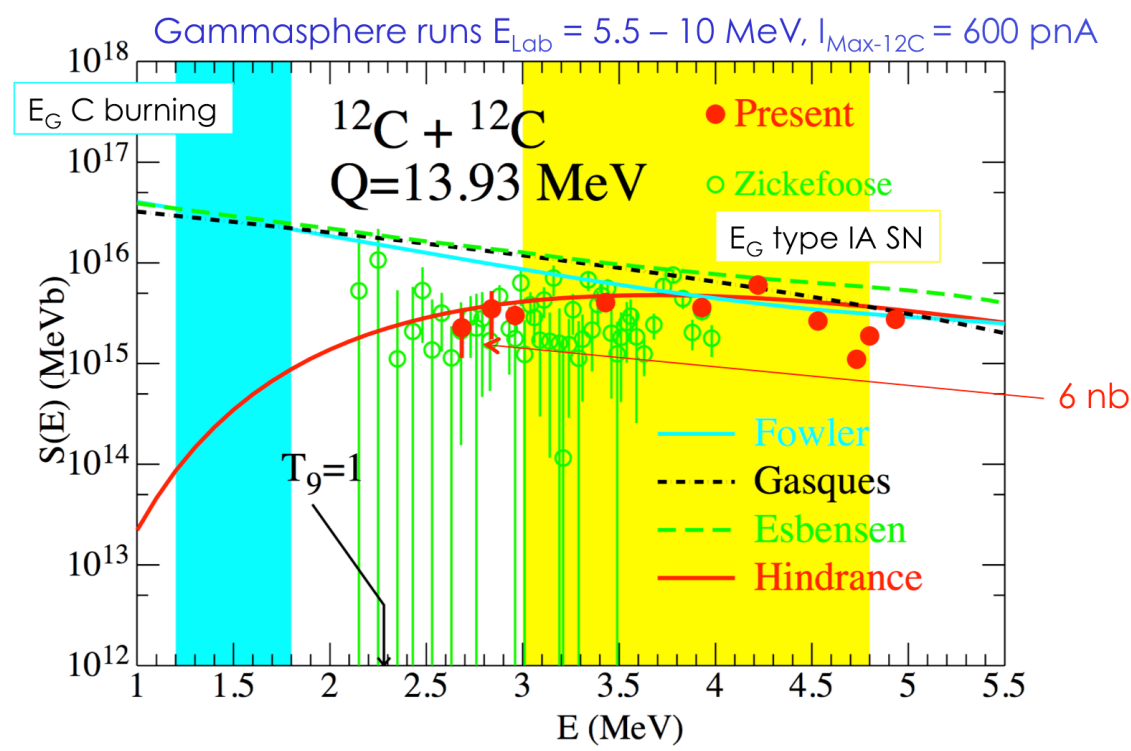

Figure 3. Red points : ${ }^{12} \mathrm{C}+{ }^{12} \mathrm{C} \mathrm{S}$ factors measured in this work. Green circles : results from ref. [12]. The blue, black, red and green dashed lines correspond to extrapolations from Fowler [13], Gasques [14], Jiang [15] and Esbensen [16].

[17] C.L. Jiang et al., Phys. Rev. Lett. 89, 052701 (2002).

[18] S. Misicu et al., Phys. Rev. Lett. 96, 112701 (2006).
[19] T. Ichikawa, K.Hagino and A. Iwamoto et al., Phys. Rev. C75, 064612 (2007) and Phys. Rev. Lett. 103, 202701 (2009). 